\title{
Detection of depression among patients with problematic alcohol use in General Out Patient setting
}

\author{
${ }^{1}$ Ogunsemi OO, ${ }^{1}$ Afe TO, ${ }^{1}$ Bodunde OT, ${ }^{2}$ Abasiubong F, ${ }^{3}$ Erinfolamin AR, \\ ${ }^{1}$ Shorunmu TO, ${ }^{1}$ Oyelekan AAA, ${ }^{4}$ Ariba AJ \\ ${ }^{\text {I}}$ Obafemi Awolowo College of Health Sciences, Olabisi Onabanjo University, Ago-Iwoye, Ogun State, Nigeria. \\ ${ }^{2}$ Department of Psychiatry, University of Uyo, Akwa ibom State, Nigeria. \\ ${ }^{3}$ Department of Psychiatry, College of Medicine, University of Lagos, Lagos state, Nigeria. \\ ${ }^{4}$ Department of family medicine, Olabisi Onabanjo University Teaching Hospital, Sagamu, Ogun State, Nigeria.
}

\begin{abstract}
Background: The nature of the relationship between depression and alcohol use is of interest to mental health professionals. Failure to detect such disorders in the hospital settings denies them access to adequate treatment. This study therefore, aimed to assess the presence of depression in patients with problematic alcohol use presenting at the General Out-patient clinic and the ability of the physicians to detect such disorders.
\end{abstract}

Methods: It was a cross-sectional descriptive study. Consecutive and voluntarily consenting patients attending the clinic over a period of eight weeks were recruited and administered the alcohol section of the patient health questionnaire. Those that met criteria for problematic alcohol use were then screened for depression using the depression section of the patient health questionnaire.

Results: Two hundred and nine (31.1\%) out of 672 that were recruited into the study met the criteria for problematic alcohol use. Sixty-five (31.1\%) of them screened positive for depression. Female gender $(p=0.000)$, unemployment $(p=0.002)$ and been married $(p=0.027)$ tend to be associated with depression. Physicians were only able to make diagnosis relating to psychopathology in $19.1 \%$ of the participants.

Conclusion: There is a need for screening for co-morbidity of depression with alcohol by primary care physicians in General Outpatient Units due to high rate of patients with such problems. Such screening will help in early intervention and consequent treatment.

\section{Introduction}

High prevalence of clinical depression in alcohol use disorders has been reported in several studies. ${ }^{1-3}$ Prevalence estimates of such co-morbidity in various settings ranged between $16-68 \%$. ${ }^{1}$ The nature of this dual relationship is of interest to mental health professionals. ${ }^{4}$ The interest is not only due to the challenge of getting an accurate diagnosis but the implications for treatment and long term prognosis. ${ }^{1,5}$ A major issue of clinical interest inherent in the co-morbidity is to delineate the predisposing or causal factor among the two disorders. Alcohol use has been postulated by some authors to be a predisposing/causal factor to depression while others are of the view that depression underlies majority of cases of alcohol use disorders. ${ }^{1,6,7}$ Till date there is no clear consensus among researchers on the nature of the relationship. Co-occurrence of depression in those with problematic use of alcohol may lead to a pattern of self-medication of depressive symptoms with alcohol, increased rate of relapse after treatment, high utilization of health services and risk of suicide ${ }^{8-10}$ Of significance is the gamut of social problems associated with alcohol which may be a factor contributing to the relationship with depression and vice-versa.

Whatever the nature of the causal relatedness, the implications for treatment are far more relevant. Failure to detect the co-morbidities by primary care or family physicians in history taking and diagnosis can bar these patients from adequate treatment and impact negatively on the prognosis. ${ }^{11-12}$ It can potentially divert treatment approaches away from the primary disorder.

Alcohol remains the commonest psychoactive substance used in Nigeria. ${ }^{13}$ Reports have documented prevalence of alcohol-related problems especially among primary care patients.$^{14-15}$ Depressive disorders have been reported to be of high prevalence in communities and primary health centers; however few studies have surveyed the prevalence of co-morbidity with alcohol in these settings. ${ }^{15-16}$

The lack of detection by clinicians in primary care setting poses significant problems in treatment, as failure to detect the co-morbidity can impact negatively on other medical disorders and deny accessibility to adequate treatment at tertiary health centers under mental health professionals. ${ }^{17-18}$ Sadly, in Nigeria, there is significant paucity of data available to family/primary physicians to highlight the need for increased suspicion and awareness among patients presenting at the Units.

In such background, increased detection of and treatment of individuals with alcohol and depressive comorbidity will be an elusive goal in our health care system. Hence, the aim of this study was to assess the 
presence of depression in patients with problematic alcohol use presenting at the General Out-patient clinic and the ability of the physician to detect such disorders.

\section{Methodology}

Setting

The study was carried out at the General Outpatient Unit of the Olabisi Onabanjo University Teaching Hospital Sagamu, Ogun State, southwest Nigeria. The General Outpatient unit doubles as a primary care and referral Centre from various health facilities from within and outside Ogun state, Nigeria. The unit runs a regular consulting clinic five days a week from Mondays to Fridays supervised by 2 consultant family physicians with the help of many residents -in-training. The clinics are usually well attended and attendees usually pay a token for registration and medication. The General Outpatient unit serves as an entry point for the Hospital and referrals are done to other units of the hospital if specialist attention is needed.

Study design and Procedure

It was a cross-sectional descriptive survey done over a period of eight weeks. Consecutive and voluntarily consenting patients attending the clinic who were aged 18 years and above were recruited from all the clinic days after their respective consultation with primary care physicians in the General Outpatient Clinic. Approval of the Ethical Committee of the Olabisi Onabanjo University Teaching Hospital was obtained.

Before commencement, the patients were briefed about the purpose of the interview before administration of the questionnaires. Patients who did not give voluntary consent and those who were not mentally and physically able to sustain an interview session were all excluded. Anonymity was assured. Trained volunteer resident doctors were used to administer the instruments and to help participants who were limited in English. Moreover, the hospital number, complaints and diagnosis made by the physicians as indicated in the case notes were noted for each participant.

The survey was done in two phases. The initial phase was used to screen all participants for problematic alcohol use and create a subset of participants who met the criteria for problematic alcohol use suitable for the next phase. Those that met the criteria for problematic alcohol use were further recruited for the second phase. The second phase involved screening for significant symptoms of depression among those with problematic alcohol use.

Study instruments

1) A well-designed socio-demographic and socio-economic questionnaire was used to elicit demographic variables such as age, sex, religion, marital status, tribe, educational status, occupation. Additionally, participants were asked to identify areas that cause much worries and concern in their lives.

\section{2) Patient Health Questionnaire}

Patient Health Questionnaire (PHQ) is a self-report scale derived from Primary-Care Evaluation of Mental Disorders. ${ }^{19}$ (PRIME-MD) which is standardized with demonstrated diagnostic performance (sensitivity of $83 \%$, specificity of $88 \%$, and positive predictive value of $89 \%$ ) for the diagnosis of any psychiatric disorder in primary health care. The instrument is designed for use in primary care and was the first to diagnose specific disorders using Diagnostic and Statistical Manual of Mental Disorders-Fourth Edition (DSM-IV). PHQ has diagnostic validity comparable to the original clinician administered and easy to use than PRIME -MD. PHQ has been variously used in different surveys in Nigeria. The section focusing on alcohol use was used to screen participants in the first phase while the section on depression was used to screen participants for depressive symptoms.

Data Analysis

Analysis was done with statistical package for science and social science version 16 . Sociodemographic and socio-economic data was presented using frequency tables and descriptive statistics. Simple cross-tabulation was also used to compare presence of clinical depression across socio-demographic characteristics of respondents.

Socio-demographic characteristics (table 1)

\section{Results}

Out of 672 that were voluntary participants in the study, a total of $209(31.1 \%)$ met the criteria for problematic alcohol use. The ages of the participants ranged from 23-90. Mean age was 54.0 \pm 19.7 years. Majority were males $(82.8 \% \mathrm{n}=173)$. Almost all the participants were employed $(95.7 \%)$ while close to threequarters were married (73.2\%). More than a quarter of them $(27.3 \%)$ had up to 12 years of education.

Presence of Depression among Participants with Alcohol Use Problems (table 2)

Out of 209 participants with alcohol use problem, 65 (31.1\%) met criteria for depressive disorder. The females $(66.7 \%)$ tend to be more depressed compared with their male counterparts $(23.1 \%)$ with alcohol 
problem $(\mathrm{x} 2=13.168 ; \mathrm{p}=0.000)$. Those that were unemployed $(88.9 \%)$ compared with those with employment $(28.0 \%)$ were significantly depressed $(\mathrm{x} 2=9.360 ; \mathrm{p}=0.002)$. The participants that were married $(36.8 \%)$ compared with those that were single, separated or divorced $(16.1 \%)$ were more depressed $(x 2=4.887 ; \mathrm{p}=$ 0.027).

Sources of stress among participants with problematic alcohol use (table 3)

Areas of high concern were with health, finances and stress at workplace. Majority of participants (95.7\%) reported being bothered about their health, about three-quarter $(76.6 \%)$ were bothered about their finances, while $73.2 \%$ were bothered about stress at their workplaces. Only a minority $(3.8 \%)$ saw their alcohol drinking as a source of stress.

Physician's recognition of psychopathology

Physicians were able to make diagnosis relating to psychopathology in $40(19.1 \%)$ of the 209 participants with alcohol problems (11.5\% neurotic disorders, $7.7 \%$ insomnia).

\section{Discussion}

Our study highlighted the prevalence of problematic use of alcohol among patients presenting at the General Outpatient unit. Complaints about alcohol use rarely forms the basis for consultation in such units. About a third (31.1\%) of the total number of participants surveyed had significant problems with alcohol as assessed by the Patient Health Questionnaire. Expectedly, none of the participants presented with complaints of alcohol at the General Outpatient Unit. Males made up more of the participants, as they are often at risk in alcohol use disorders. ${ }^{20}$ Apart from the sex distribution, alcohol-related problems were more prevalent in those who were married, unemployed and with lower years of educational attainment. The socio-demographic distribution is similar to the findings of an earlier study in East Africa. ${ }^{1}$

The occurrence of clinically significant depression among participants who reported problems with alcohol use in our study was $31.1 \%$. Prior studies have often reported an association with depression with greater risk with increasing use of alcoholic beverage. ${ }^{3,6}$ The rate of depression among our participants falls within the usual range of $16-68 \%$ as found in most studies. ${ }^{21}$ Comparatively, the rate of depression among our participants was much higher than the estimated rate among the general populace in Nigeria. ${ }^{22}$ Our finding is consistent with earlier studies that reported clinically significant depression as a common co-morbidity among patients with alcohol-use problems. More obvious from our study is that such co-morbidity is quite prevalent (about a third) among patients presenting at the General Outpatients Units.

Across socio-demographic variables, depression was significantly higher in females (66.7\%) than males $((23.1 \%)$. Although it is known that females have a lower risk developing alcohol problems, females who develop such alcohol problems have a higher risk of developing psychopathology than males who have problems with alcohol. ${ }^{23}$ Previous surveys among subjects with alcohol-related problems, reported higher rate of depression among females and they are more prone to use Government Health Facilities than males with similar problem. ${ }^{23}$ Those who were married had significantly higher rate of depression than those who were single or never married(36.8\%). This may be due to conflicts in marital relationship that can result due to alcohol abuse with consequent negative impact on mental health. ${ }^{24}$ Also, the unemployed had higher rate of depression than those with employment. Understandably, unemployment can give rise to socio-economic difficulties, and abusing alcohol might be seen as some as a way of reliving such difficulties.

Importantly, participants related socio-economic issues that were very stressful to their lives. Health worries, finances and stress at workplace were notable areas of much concern. Among all these social issues, concern about their health was quite predominant. Obviously, Social stress has long been an associated consequence of problematic use of alcohol. Problematic alcohol use creates difficulties within the individual's social milieu, and has been linked to social stress and problems in all these areas of concern as reported by the participants. ${ }^{25}$ Alcohol abuse fuels a web of interlinked social consequences to the individual and the society at large. These social concerns should be explored in patients with alcohol problems as they may be significant factors to the co-morbidity with depression.

Attending physicians diagnosed Neurotic disorders among $11.5 \%$ of participants and insomnia in $7.7 \%$ of participants. Not surprisingly, however, there was no diagnosis of alcohol use problems with depressive comorbidity made by the attending physicians among the participants in our study. The poor rate of detection could be attributable to the fact that patients rarely volunteer information about their use of alcoholic beverages probably due to avoidance of social stigma and self-denial. They are more likely to present when there is significant major health and social issues. ${ }^{17,26}$ Additionally, most physicians rarely screen for alcohol use problems and associated psychological morbidities due to a variety of factors such as insufficient time for such interviews, diagnostic methods, lack and paucity of knowledge on alcohol use disorders and screening instruments in primary care settings., ${ }^{17,27}$

The failure to detect alcohol-related co-morbidity in General Outpatient Units by attending physicians creates a large void that excludes a significant number of patients who could benefit from specialist treatment 
services and early intervention. Increasingly, studies have favored early detection and intervention in treatment of alcohol-related co-morbidities which can be hampered by the low rate of recognition by attending healthcare workers and physicians in primary care settings. Although we acknowledge the limitation of study in not exploring other multiple factors in relationship with the co-morbidity and tendency for patients to under-report, however, our study brings to the fore the need to explore socio-economic issues when attending to patients with significant alcohol-related co-morbidity with depression.

Conclusively, there is a need for screening for co-morbidity of depression with alcohol by primary care physicians in General Outpatient Units due to high rate of patients with such problems. Furthermore, screening will help in early intervention and consequent treatment. Use of self-report instruments such as the PHQ, can help family physicians working in General outpatient units to make early detection and treatment.

\section{References}

[1]. Kuria MW, Ndetei DM, Obot SI et al. The Association between Alcohol Dependence and Depression before and after Treatment for Alcohol Dependence. International Scholarly Research Network ISRN Psychiatry, 2012 (2012), 482802.

[2]. Grant BF and Harford TC. Co-morbidity between DSM-IV alcohol use disorders and major depression: Results of a national survey. Drug and Alcohol Dependence, 1995; 39(3): 197-206.

[3]. Boden JM and Fergusson DM. Alcohol and Depression. Addiction, 2011; 106(5):906-914

[4]. Berner P, Lesch OM and Walter H. Alcohol and Depression. Psychopathology 1986;19(suppl 2):177-183

[5]. Mueller TI, Lavori PW, Keller MB et al. Prognostic effect of the variable course of alcoholism on the 10-year course of depression. American Journal of Psychiatry, 2004; 151 (5):701-706.

[6]. Dackis, CA, Gold MS, Pottash ALC et al. Evaluating depression in alcoholics. Psychiatry Research 1986:17(2):105-9.

[7]. Flensborg-Madsen T, Mortensen EL, Knop J et al. "Co morbidity and temporal ordering of alcohol use disorders and other psychiatric disorders: results from a Danish register-based study," Comprehensive Psychiatry, 2009 : 50, (4): 307-314.

[8]. Khantzian EJ. The self medication hypothesis of addictive disorders: Focus on heroin and cocaine dependence. American Journal of Psychiatry 1986; 142(11):1259-1264.

[9]. Pettinati HM. Antidepressant treatment of co-occurring depression and alcohol dependence. Biological Psychiatry 2004; 56(10): 785-792.

[10]. Conner KR. and Duberstein PR. Predisposing and precipitating factors for suicide among alcoholics: empirical review and conceptual integration. Alcoholism Clinical and Experimental Research 2004:28 (s1), 6S-17S

[11]. Angold A, Costello EJ, Erkanli A. "Comorbidity," Journal of Child Psychology and Psychiatry, 1999: 40 (01): 57-87.

[12]. Grant BF, Stinson FS, Dawson AD. Prevalence and Co-occurrence of Substance Use of Disorders and Independent Mood and Anxiety Disorders: Results from the National Epidemiologic Survey on Alcohol and Related Conditions. Arch Gen Psychiatry: 2004;61(8):807-816.

[13]. Gureje O, Degenhardt L, Olley B et al. A descriptive epidemiology of substance use and substance use disorders in Nigeria during the early 21 st century. Drug and Alcohol Dependence 2007; 91(1):1-9.

[14]. Abiodun OA. Alcohol-related problems in primary care patients in Nigeria. Acta Psychiatrica Scandinavica 1996;93(4):235-9.

[15]. Gureje O, Obikoya B, Ikuesan BA. Alcohol abuse and dependence in urban primary care clinic in Nigeria. Drug Alcohol Dependence .1992;30:163-7.

[16]. Gureje O, Lasebikan VO, Kola L et al. Lifetime and 12-month prevalence of mental disorders in the Nigerian Survey of Mental Health and Well-Being. British Journal of Psychiatry 2006: 188:465-471.

[17]. Ogunsemi OO, Oluwole FA, Abasiubong F et al. Detection of mental disorders with the Patient Health Questionnaire in primary care settings in Nigeria. Mental Illness 2010:2 (1):e10

[18]. Schuckit MA. Alcohol-use disorders. Lancet 2009; 373:492-501.

[19]. Spitzer RL, Kroenke K, Williams JB. Validation and utility of a self-report version of PRIME-MD, the PHQ primary care study. J Am Med Assoc 1999; 282:1737-44.

[20]. Wilsnack RW, Wilsnack SC, Kristjanson AF et al. Gender and alcohol consumption: patterns from the multinational GENACIS project. Addiction, 2009; 104(9): 1487-1500.

[21]. Halikas JA, Herzog MA, Mirassou MM et al. Psychiatric diagnosis among female alcoholics. Currents in Alcoholism, 1980; 8: 281-291.

[22]. Gureje O, Uwakwe R, Oladeji B et al. Depression in adult Nigerians: results from the Nigerian Survey of Mental Health and Wellbeing. J Affect Disord. 2010;120(1):158-64.

[23]. Miller NS, Klamen D; Hoffmann NG et al. Prevalence of Depression and Alcohol and Other Drug Dependence in Addictions Treatment Populations. J Psychoactive Drugs. 1996; 28(2):111-124.

[24]. Burket TR. The Economic Impact of Alcohol Abuse and Alcoholism. Public Heath Repors. 1988; 103(6):564-568.

[25]. World Health Organisation. Global Status Report on Alcohol 2004: Social problems associated with Alcohol Use-Part 1. Pp 59-64. Published by World Health Organisation, Department of Mental Health and Substance Use Geneva.

[26]. Mitchell AJ, Meader N, Bird V et al. Clinical recognition and recording of alcohol disorders by clinicians in primary and secondary care: meta-analysis. BJP 2012; 20 193-100.

[27]. Abiodun OA. A study of mental morbidity among primary care patients in Nigeria. Compr Psychiatry. 1993; 34(1):10-3.

Table 1;Socio-demographic characteristics of Patients with Problematic Alcohol Use

\begin{tabular}{|l|c|c|}
\hline Variables & Frequency & Percentage \\
\hline AGES & & 26.8 \\
$\mathbf{2 0 - 4 0}$ & 56 & 31.1 \\
$\mathbf{4 1 - 6 1}$ & 65 & 34.9 \\
$\mathbf{6 2 - 8 2}$ & 73 & 7.2 \\
$\mathbf{> 8 2}$ & 15 & 82.8 \\
SEX & & \\
MALE & 173 & \\
\hline
\end{tabular}


Detection of depression among patients with problematic alcohol use in General Out Patient setting

\begin{tabular}{|l|c|c|}
\hline FEMALE & 36 & 17.3 \\
\hline MARITAL STATUS & & \\
\hline SINGLE/NEVER MARRIED & 56 & 26.8 \\
\hline MARRIED & 153 & 73.2 \\
EMPLOYMENT STATUS & & \\
EMPLOYMENT & 200 & 95.7 \\
UNEMPLOYMENT & 9 & 4.3 \\
EDUCATION & & \\
S12 YEARS & 152 & 72.7 \\
$>12$ YEARS & 57 & 27.3 \\
\hline
\end{tabular}

Table 2: Socio-demograhic characteristics and depression among participants with problematic alcohol use

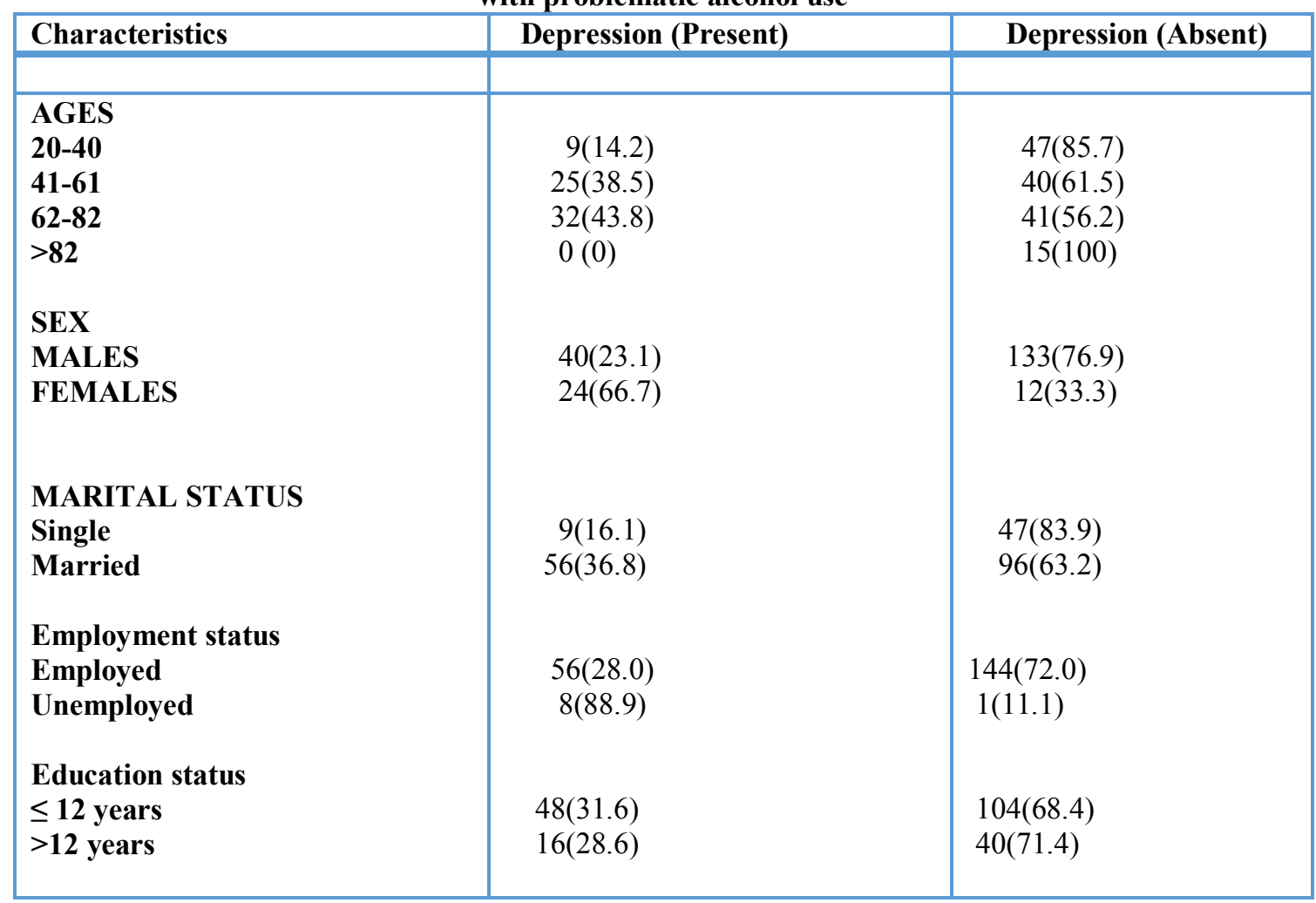

Table 3: Sources of stress among participants with problematic alcohol use

\begin{tabular}{|l|c|c|}
\hline \multicolumn{1}{|c|}{ Worries } & Bothered (\%) & NOT Bothered (\%) \\
\hline About Health & $200(95.7)$ & $9(4.3)$ \\
About work & $153(73.2)$ & $56(26.8)$ \\
About finances & $160(76.6)$ & $49(23.4)$ \\
About my drinking & $15(7.2)$ & $194(92.8)$ \\
& $8(3.8)$ & $201(96.2)$ \\
\hline
\end{tabular}

